\title{
Grain formation in post-shocked wind collisions of massive binary systems
}

\author{
Diego Falceta-Gonçalves, Vera Jatenco-Pereira, and Zulema Abraham \\ Instituto de Astronomia, Geofísica e Ciências Atmosféricas, USP, \\ Rua do Matão 1226, 05508-090 São Paulo, Brasil
}

\begin{abstract}
Massive binary star systems are not uncommon, and ncither the supersonic collision of their winds. In the present work we study these shocks and the further consequences on wind structure. The post-shock gas is a warm and high-density environment, which allows dust to form and grow. We show that this growth is fast, of just a few hours. An application to $\eta$ Car shows that, probably, the decline of X-rays fluxes observed in its light curve is the consequence of its high absorption in periodic dust formation events, at periastron passage.
\end{abstract}

\section{Introduction}

Many massive binary systems are known, and almost all present high-energy fluxes, not originated in the stars, but around them, indicating the presence of colliding winds. The source of observed high X-ray fluxes should be a hot and dense gas, generated by shocks around these objects (Zhekov \& Skinner 2000). At high temperature and density, free-free emission in X-rays becomes highly important (Usov 1992; Ishibashi et al. 1999). X-ray emissions associated with interacting winds were observed on many objects (Williams et al. 1990; Corcoran et al. 2001; Thaller et al. 2001). Grains also may be formed and grow on postshocked gases (e.g., Williams et al. 1987, 1990; Monnier et al. 2001; Williams et al. and Tuthill et al. in these Proceedings), when the heated gas loses high amount of energy, mainly by Bremsstrahlung, cools and becomes denser. Recent observations in the IR supply this idea, showing episodic dust formation events in these regions (Williams et al. 1990; Marchenko et al. 1999; Harries et al. 2000). Some binary systems present, periodically, a sudden decrease of X-ray emission (Ishibashi et al. 1999). A fast dust formation and growth event in these regions due to the shocked winds might provoke a considerable increase in extinction explaining these features of the light curves. This could cause the temporary reduction on X-rays and UV fluxes, which may increase a posteriori with the evaporation of the particles, or even by the expansion of the dust shell.

\section{Model and results: an application to $\boldsymbol{\eta}$ Carinae}

The model assumes the colliding winds of two massive stars $A$ and $B$, which have mass loss rates $\dot{M}_{\mathrm{A}}$ and $\dot{M}_{\mathrm{B}}$, and wind velocities $v_{\mathrm{A}}$ and $v_{\mathrm{B}}$, respectively, orbiting each other with period $P$, eccentricity $e$ and separation $D$ at periastron. 
We divide the shock evolution in four parts: $(i)$ the instantaneous collision, neglecting radiative losses; $(i i)$ the gas cooling at post-shock; (iii) the formation of dust and its growth; and (iv) the evolution of the dust shell, and its consequence to the light curve observed. The basic physics of the shock is very well known, and described by the hydrodynamics equations of mass continuity, fluid momentum and energy. The growth and evaporation rates of the dust particles are given by the classic nucleation theory.

$\eta$ Car is a super massive star, supposed to be a LBV class star, and also is supposed to have a companion, probably a massive WR or OB star (Damineli et al. 2000). The system total mass, inferred by luminosity, is $\sim 120 \mathrm{M}_{\odot}, \eta$ Car A is responsible for major of this mass and $\eta$ Car B should have typical WR stellar winds, $\dot{M}_{\mathrm{B}} \approx 10^{-5} \mathrm{M}_{\odot} \mathrm{yr}^{-1}$ and $v_{\mathrm{B}}^{\infty} \approx 3 \times 10^{3} \mathrm{~km} \mathrm{~s}^{-1}$. Observations indicate that $\eta$ Car A should have $\dot{M}_{\mathrm{A}} \approx 10^{-3} \mathrm{M}_{\odot} \mathrm{yr}^{-1}$ and $v_{\mathrm{A}}^{\infty} \approx 500 \mathrm{~km} \mathrm{~s}^{-1}$ (Hillier et al. 2001; Damineli et al. 2000). For the above parameters and $\dot{M}=5 \times 10^{-4} \mathrm{M}_{\odot} \mathrm{yr}^{-1}$ and $e \simeq 0.8$, it was possible to reproduce its observed light curve.

\section{Conclusions}

In this work we present a model for colliding winds, where we determined the changes in physical parameters, like density, temperature and pressure in the post-shock. This gas, cooled and denser after shock, can be the site for grains formation. The model was applied to $\eta$ Car which could explain: $(i)$ the rapid decline on fluxes observed periodically; and $(i i)$ the slight decrease in opacity considering the expansion of the dust with the wind. This model agrees better with observations for $e \simeq 0.8$, and a mass loss rate of the primary star of about $3-5 \times 10^{-4} \mathrm{M}_{\odot} \mathrm{yr}^{-1}$.

Acknowledgments. The authors thank the Brazilian agencies CNPq, CAPES and FAPESP for support, as well as the project PRONEX (41.96.0908.00).

\section{References}

Corcoran, M.F., Ishibashi, K., Swank, J.H., Petre, R. 2001, ApJ 547, 1034

Damineli, A., Kaufer, A., Wolf, B., Stahl, O., Lopes, D.F., de Araújo, F.X. 2000, ApJ 528, 101

Harries, T.J., Babler, B.L., Fox, G.K. 2000, A\&A 361, 273

Hillier, D.J., Davidson, K., Ishibashi, K., Gull, T.R. 2001, ApJ 553, 837

Ishibashi, K., Corcoran, M.F., Davidson, K., Swank, J.H., Petre, R., Drake, S.A., Damineli, A., White, S. 1999, ApJ 524, 983

Marchenko, S.V., Moffat, A.F.J., Grosdidier, Y. 1999, ApJ , 522, 433.

Monnier, J.D., Tuthill, P.G., Danchi, W.C. 2001, A\&AS 199, 608

Thaller, M.L., Gies, D.R., Fullerton, A.W., Kaper, L., Wiemker, R. 2001, ApJ 554, 1070.

Usov, V.V. 1992, ApJ 389, 635

Williams, P.M., van der Hucht, K.A., Thé, P.S. 1987, A\&A 182, 91

Williams, P.M., van der Hucht, K.A., Pollock, A.M.T., Florkowski, D.R., van der Woerd, H., Wamsteker, W.M. 1990, MNRAS 243, 662

Zhekov, S.A., Skinner, S.L. 2000, ApJ 538, 808 